\title{
EL DESARROLLO DE LAS COMPETENCIAS SOCIO-PROFESIONALES A TRAVÉS DEL PORTAFOLIO EN PSICOPEDAGOGÍA
}

\author{
Natalia González Fernández \\ Universidad de Cantabria
}

\section{RESUMEN}

El presente artículo está basado en la experiencia de uso del portafolio como herramienta de enseñanza, aprendizaje y evaluación de los contenidos y competencias socio-profesionales necesarias en la asignatura de "Diagnóstico en Educación". Esta asignatura se enmarca dentro de los estudios superiores de la licenciatura en Psicopedagogía.

Frente a modelos tradicionales de enseñanza-aprendizaje y evaluación basados en el temario y el dominio de contenidos por parte de los estudiantes, el nuevo modelo de formación de la asignatura, se articula sobre la definición y priorización de competencias y diseño de actividades cooperativas e individuales que posibiliten el desarrollo de dichas competencias profesionales

Finalmente se presentan análisis de los resultados derivados de las reflexiones y valoraciones de los estudiantes implicados en la experiencia obtenidos en una encuesta abierta, demostrando que el portafolio es un método eficaz de enseñanza, aprendizaje y evaluación en la formación universitaria.

PALABRAS CLAVE: Portafolio, EEES, Competencias Profesionales.

\section{ABSTRACT}

This paper is based on the experience of developing a methodology the portfolio as a tool of teaching, learning and evaluation to the professional competences and contents in Educational Diagnostic. This subject is framed inside de upper studies of the degree in Sciences of Education, Psicopedagogy section.

Facing traditional models of teaching, learning and evaluation based on the syllabus and in the control of student,s know-how, the new subject formation model is articulated on the definition and priority of professional competences and design of cooperative and individual activities that enable the development of these professional competences.

Finally presents analysis of the results derived from the reflections and valuations of the students implied in the experience obtained in an open sur- 
vey appear, demonstrating that portafolio is an effective method of education, learning and evaluation in the university formation.

KEY WORDS: Portfolio, European Space in Higher Education, professional Competences.

\section{FUNDAMENTACIÓN TEÓRICA: EL PORTAFOLIO COMO PROPUESTA DE INNOVACIÓN EN PSICOPEDAGOGÍA}

El paradigma formativo en el Espacio Europeo de Educación Superior enfatiza un modelo de formación centrado en el trabajo y aprendizaje del estudiante, mediante el desarrollo de competencias generales y específicas, que posibilitan un aprendizaje continuo a lo largo de toda la vida y una adaptación a la variedad y pluralidad cultural de nuestro contexto europeo actual.

Según este lenguaje e impulso, el trabajo en grupo, la formación de equipos de aprendizaje, el desarrollo de actitudes de cooperación, etc, constituyen una variable importante de formación básica en la Enseñanza Superior, de cara a una eficaz integración de los futuros titulados en el ámbito laboral. Por tanto, no se trata tan solo de un cambio administrativo del sistema de créditos (ECTS), supone un replanteamiento pedagógico global y de raíz. Aportaciones recientes de Colás y De Pablos (2005) y González, y Wagennar, (2003) proponen experiencias de adaptación de modelos didácticos y organizativos a los ECTS.

En este proceso de convergencia la actividad docente adquiere nuevos enfoques y es necesario no solo que se actualice, sino que se forme y tome decisiones en torno a los prodecimientos y recursos a utilizar en las diferentes fases de su planificación o programación anual. De Miguel et al (2006) sintetiza una serie de métodos didácticos junto con su finalidad que pueden servirnos de guía y reflexión, tales como los expuestos en el siguiente cuadro.

\section{Cuadro 1}

\begin{tabular}{|l|l|}
\hline MÉTODO & FINALIDAD \\
\hline Método Expositivo / Lección Magistral & $\begin{array}{l}\text { Transmitir conocimientos y activar pro- } \\
\text { cesos cognitivos en el estudiante }\end{array}$ \\
\hline Estudio de Casos & $\begin{array}{l}\text { Adquisición de aprendizajes mediante } \\
\text { el análisis de casos reales o simulados }\end{array}$ \\
\hline Resolución de Ejercicios o Problemas & $\begin{array}{l}\text { Ejercitar, ensayar y poner en práctica los } \\
\text { conocimientos previos }\end{array}$ \\
\hline Aprendizaje Basado en Problemas & $\begin{array}{l}\text { Desarrollar aprendizajes activos a través } \\
\text { de la resolución de problemas }\end{array}$ \\
\hline Aprendizaje Orientado a Proyectos & $\begin{array}{l}\text { Realización de un proyecto para la reso- } \\
\text { lución de un problema, aplicando habi- } \\
\text { lidades y conocimientos adquiridos }\end{array}$ \\
\hline Aprendizaje Cooperativo & $\begin{array}{l}\text { Desarrollar aprendizajes activos y signi- } \\
\text { ficativos de forma cooperativa }\end{array}$ \\
\hline Contrato de Aprendizaje & \begin{tabular}{l} 
Desarrollar el aprendizaje autónomo \\
\hline
\end{tabular}
\end{tabular}


Nosotros añadimos a la clasificación de los citados autores, el Método del Portafolio, con el que pretendemos potenciar y desarrollar no solo contenidos, sino procedimientos y actitudes. El portafolio se puede definir de múltiples modos. Sintéticamente se puede decir, que es una recopilación intencionada de información generada y elaborada por un estudiante para demostrar lo que ha aprendido teórica y prácticamente y las competencias utilizadas para resolver un problema o proyecto.

El portafolio hace referencia a un modo alternativo de organizar los procesos cognitivos que se han de provocar en un proceso de enseñanza aprendizaje tanto dentro como fuera del aula. Es decir, se trata con su implementación de superar determinadas "lagunas" generadas con la aplicación exclusiva de técnicas tradicionales de aprendizaje grupal, interesadas más por resultados que por rendimientos, por responsabilidades grupales más que individuales, por grupos homogéneos más que heterogéneos, líderes únicos en vez de liderazgos compartidos, etc.

Requiere una programación más centrada en el aprendizaje que en la enseñanza, en la que tanto el docente como el estudiante han de planificar al detalle las actividades que han de desarrollar en cada sesión de la asignatura, tanto dentro como fuera del aula. Por tanto, si el profesor no puede hacer lo que tenía previsto, la planificación no se resiente tanto como si el estudiante no realiza su tarea. Para Cowan (1998), la tarea del profesor consiste en crear situaciones de las que el estudiante no pueda escapar sin haber aprendido.

Las ventajas del uso de las técnicas del portafolio en educación contrastadas en numerosos trabajos de investigación anteriores, y que alientan a seguir mejorando y evaluando sus consecuencias y trascendencia real en el aprendizaje, han sido resumidas por Lyons (1995); resaltamos aquí algunas tales como, el aprendizaje directo de actitudes y valores, la mejora de la motivación, la generación de comunidades comprensivas, el desarrollo de una mayor crítica constructiva, etc.

A pesar de todas las ventajas que tiene esta metodología, debemos ser conscientes de que nos vamos a encontrar con dificultades como éstas: espacios inadecuados para el desarrollo de trabajos en grupo, el tiempo para corregir y evaluar se incrementa, cambio en el sistema de evaluación: continua / final, absentismo escolar ante los exámenes, falta de experiencia del profesorado, individualismo del profesorado, excesivo número de estudiantes por aula, falta de recursos, etc.

En este trabajo concretamente nos hemos centrado en analizar el grado de desarrollo de determinadas competencias socio-profesionales en los estudiantes de Psicopedagogía, desarrollando los contenidos de la asignatura de "Diagnóstico en Educación" a través de una metodología cooperativa, autónoma, en general dialéctica y constructivista y, en ocasiones, expositiva-magistral. Las competencias socio profesionales generales trabajadas, observadas y valoradas en esta propuesta de innovación para la asignatura antes referida, a cuya programación le hemos dado un fuerte componente experiencial, basado en el diseño y puesta en práctica de una serie de actividades reales y cercanas a su entorno laboral inmediato, han sido estas diez:

- Habilidades comunicativas: desarrollo de capacidad verbal, en cuanto a comprender, explicar, preguntar y responder, debatir, utilizar correctamente terminología de la asignatura, etc.

- Capacidad de Síntesis, para extraer lo fundamental prescindiendo de lo accesorio. 
- Análisis y reflexión: capacidad para deliberar, pensar, repasar, reconsiderar y madurar una idea antes de tomar una decisión.

- Crítica Constructiva: capacidad para posicionarse ante las opuestas o diferentes intervenciones de los compañeros, sin anularlos, ni imponerse, sino aportando su visión y enriqueciendo el resultado.

- Implicación: aportar sugerencias e ideas en las diferentes actividades, comprometiéndose en su desarrollo hasta el final.

- Autonomía: resolver actividades utilizando recursos propios sin recurrir a la ayuda inmediata del docente.

- Creatividad: diseñar o generar recursos didácticos (debate, rol-playing, dramatización, etc., con ingenio, novedad y aplicabilidad)

- Iniciativa: buscar soluciones indagando "motu proprio", sin ser motivado o apoyado exteriormente.

- Autoplanificación: gestión del propio tiempo

- Voluntad: capacidad de constancia y esfuerzo continuado en el desarrollo de actividades.

Existen diversas técnicas a través de las cuáles el profesorado puede diseñar las tareas a realizar, así como diversas formas de evaluar los resultados. En esta comunicación, por razones de espacio, nos limitaremos a presentar las técnicas más conocidas, ya que son las que hemos utilizado en nuestra experiencia:

- Técnica de "puzzle" o "rompecabezas" de Aronson (1978): El profesor divide o fragmenta la materia a estudiar en tantas secciones como miembros compongan el grupo de trabajo. Cada estudiante se encargará de leer su texto. Posteriormente se reúnen en "grupos de expertos" con compañeros que han leído el mismo texto, para compartir la información. Finalmente, todos vuelven a sus grupos de origen para elaborar conjuntamente el material.

- Técnica de "Grupo de investigación" de Sharan (1980): Los estudiantes escogen subtemas de la materia que tienen que estudiar en clase. Cada grupo divide los subtemas en tareas individuales dentro del grupo. Los estudiantes investigan los subtemas juntos y después presentan los resultados a toda la clase.

- Contrato de Aprendizaje: Ios estudiantes eligen los trabajos o las tareas que realizan individualmente o en pequeños grupos a fin de ejercitar conocimientos y usar competencias. Quedan plasmados por escrito y pretenden integrar variables intelectuales, sociales y creativas.

En todo momento hemos querido provocar la reflexión, la creatividad y la crítica constructiva de los estudiantes, pero sobre todo su implicación a la hora ayudarnos a decidir consensuada y justificadamente, si realmente el esfuerzo humano y material a afrontar optando por una metodología alternativa, merece la pena. Por los resultados obtenidos en este estudio deducimos que el cambio es, no solo positivo, sino necesario en nuestra titulación. 


\section{PLANTEAMIENTO Y DISEÑO METODOLÓGICO}

\section{PARTICIPANTES}

En la investigación participaron 52 de los 88 estudiantes matriculados en el primer curso de la titulación de $2^{\circ}$ ciclo de Psicopedagogía de la Universidad de Cantabria. Este curso 2005-2006 hemos acusado una mayor asistencia a clase, a pesar de seguir contando con una población elevada de estudiantes que al poseer ya una titulación universitaria de primer ciclo están integrados en el ámbito laboral en ramas como la docencia, la asistencia social, escuelas de ocio y tiempo libre, etc.

\section{OBJETIVOS}

Este trabajo de innovación que tiene como finalidad última comprobar el potencial didáctico del portafolio en la enseñanza universitaria, se plantea más específicamente cubrir determinados objetivos de índole científica, instrumental y aplicada. Estos objetivos se concretan en:

1. Diseñar un protocolo dirigido y adaptado a los estudiantes universitarios para la creación de un portafolio. Entendiendo que la clave del éxito de la experiencia está en dejar secuenciada y muy clara la estructura y organización del portafolio.

2. Identificar las dificultades y resistencias que conlleva el cambio metodológico.

3. Observar su utilidad y aprovechamiento en la concienciación del estudiante sobre el desarrollo de las competencias establecidas.

4. Comparar el grado de desarrollo de competencias socio-profesionales en los estudiantes a través del aprendizaje tradicional y a través del portafolio.

5. Detectar indicadores de cambios y progresos en las competencias, a través del análisis continuo del portafolio.

6. Reconocer las ventajas y desventajas de la metodología del Portafolio, desde el punto de vista de los estudiantes implicados.

7. Idear procedimientos objetivos de evaluación del estudiante en base al portafolio, a través de la entrega y reflexión de evidencias de aprendizaje.

\section{HIPÓTESIS}

- Tras la experiencia del curso 2005-2006, los estudiantes tienen actitudes y opiniones más positivas sobre el potencial pedagógico del portafolio que sobre el aprendizaje tradicional.

- La participación e implicación entre los estudiantes y los estudiantes con el docente son más activas y fluidas a través del portafolio.

- El dominio de los contenidos de la asignatura es mayor, significativamente hablando, debido a las conexiones que se realizan entre los conceptos teóricos y la resolución de problemas y casos reales.

- Los estudiantes aprenden a aprender ayudando a sus compañeros de equipo, generando cohesión grupal. 


\section{METODOLOGÍA}

La experimentación de la innovación metodológica sigue las siguientes fases:

- Diseño de una guía destinada a los estudiantes matriculados en la asignatura de "Diagnóstico en Educación", orientada a la elaboración del portafolio.

- Periodo de experimentación del portafolio a través de actividades basadas en Aprendizaje Cooperativo, Aprendizaje Basado en Problemas, Proyectos de Investigación, etc.

- Proceso de reflexión, evaluación y autoevaluación continuo.

- Recogida de datos a través de preguntas reflexivas abiertas.

- Análisis de resultados y conclusiones.

En la $1^{\text {a }}$ fase, comenzamos por argumentar sobre cuestiones conceptuales cómo: ¿por qué utilizar el método del portafolio?, ¿qué es un portafolio?, ¿cuáles son las ventajas y desventajas del portafolio?, ¿cómo se elabora?, ¿qué es una competencia?, ¿y una evidencia?, ¿qué tipo de competencias se van a desarrollar en esta asignatura?

En la $2^{\circ}$ fase, experimentamos la puesta en marcha de un calendario de sesiones teórico-prácticas, pautadas metodológicamente, con criterios, temporalizaciones, agrupaciones, materiales y dinámicas de reflexión y autoevaluación previamente definidas. Las resistencias iniciales y la necesidad de realizar un feed-back continuo del proceso, nos hizo considerar la pertinencia de trabajar alternativamente actividades de carácter autónomo junto con actividades cooperativas en grupos de hasta 6 personas, con una única asignatura troncal del $2^{\circ}$ cuatrimestre de la titulación de Psicopedagogía.

En la $3^{\text {a }}$ fase iniciamos, desde el comienzo de las primeras actividades, un proceso de investigación evaluativo adoptando la forma de diseño de grupo único, supervisando y evaluando intrasujeto, el desarrollo de las competencias establecidas en la guía del Portafolio. La evaluación de la innovación sigue una secuencia polietápica, iniciándose la experiencia y manteniéndose a lo largo de la misma, una evaluación cualitativa. A dicha evaluación se dedica este artículo, con el objeto de detectar aquellas variables y dimensiones de aprendizaje que potencia el portafolio desde la perspectiva de los propios implicados. Según Klenowski (2004) las autovaloraciones aportan puntos de vista sobre pensamientos, reflexiones, sensaciones y argumentaciones de la experiencia vivida por el estudiante. Posteriormente realizamos un análisis de contenido de todos los documentos redactados.

En la $4^{\text {a }}$ fase, a los textos se les aplica una análisis de datos cualitativo que incluye las siguientes fases: Análisis Exploratorio, para identificar dimensiones discursivas generales. Análisis Descriptivo, para matizar y concretar en categorías cada una de las dimensiones anteriores. Análisis Confirmatorio, de segmentos de textos identificados en códigos correspondientes a las categorías. Para las tareas automáticas de análisis se utiliza el programa Nud-Ist (V.5.0)

\section{ANÁLISIS E INTERPRETACIÓN DE RESULTADOS}

Presentamos en primer lugar los resultados correspondientes al análisis descriptivo, mostrando las diferencias entre la metodología del aprendizaje tradicional y el aprendizaje a través del Portafolio. Llegaron a más de ciento cincuenta los aspectos 
pedagógicos mencionados sobre el portafolio. A continuación presentamos una muestra de ellos:

- "El portafolio te hace investigar".

- "El portafolio favorece el pensamiento crítico del estudiante".

- "El portafolio es un instrumento de evaluación continua".

- "El portafolio ayuda a vincular la teoría con la práctica".

- "El portafolio favorece un aprendizaje más valioso, es creado por uno mismo, lo cual ayuda a reflexionar sobre lo que se hace".

- "Con el portafolio se obtiene un feed-back continuo que ayuda a mejorar".

- "Con el portafolio se desarrollan las competencias y roles específicos de nuestra profesión".

- "El portafolios posibilita una práctica más activa en la asignatura"

- "El portafolios ayuda a desarrollar autonomía"

- El portafolios es útil porque se asimilan significativamente los contenidos de la asignatura".

La técnica utilizada nos permite elaborar dimensiones más generales que sistematizan grupos de variables próximas conceptualmente, hemos deducido las siguientes dimensiones: estilo de aprendizaje que fomenta y facilita, desarrollo de competencias, conexión teoría práctica-aprendizaje, participación, supervisión y evaluación.

De cada una de estas dimensiones nacen categorías y códigos que las identifican, pero su enumeración nos parece irrelevante de ahí que no se incluyan en este artículo.

\section{Estilos de Aprendizaje}

En líneas generales el portafolio ha supuesto para los estudiantes una amalgama de esfuerzos, por un lado para fusionar dos estilos de aprendizaje: autónomo y cooperativo, puesto que se le propuso por un lado asimilar y profundizar individual y autónomamente sobre determinados contenidos de la asignatura y por otro, integrarse para aportar y enriquecerse en trabajos de grupos formales cooperativos.

El conocimiento del estilo de aprendizaje predominante es una herramienta docente muy útil para adaptar el estilo de enseñanza del profesor de cara a mejorar el rendimiento académico (Duda y Rely, 1990). A la vez los estudiantes pueden planificar el aprendizaje según sus estilos evitando bloqueos y optimizando resultados (Alonso, 1992).

En este sentido el portafolio se convierte en una buena herramienta para desarrollar tanto una responsabilidad de aprendizaje individual como grupal. Conformando un perfil del estudiante más acorde con los requerimientos del nuevo EEES. Expresiones manifestadas en los textos amplían a la vez que matizan el valor del portafolios como herramienta que potencia la autoplanificación del aprendizaje y el trabajo cooperativo a la vez que autónomo:

"Es más fácil estudiar porque los apuntes se trabajan conjuntamente entre compañeros y profesor y el aprendizaje es significativo" 
"Implica más reflexión y motiva hacia el aprendizaje"

"Impulsa a buscar tus propias inquietudes e intereses"

"Fomenta el afán de auto superación"

"Es un trabajo continuo, día a día"

(Estudiantes de $1^{\circ}$ de Psicopedagogía)

\section{Desarrollo de competencias}

El análisis efectuado recaba información sobre los aspectos relevantes que caracterizan al portafolio como herramienta idónea para el desarrollo de competencias socio-profesionales. Los estudiantes manifiestan haber alcanzado sus expectativas en relación a la adquisición o desarrollo de competencias de forma más clara, concisa y sistemática a través del portafolio y no tanto con una metodología tradicional:

"Se ponen en juego competencias socio-profesionales, que con una metodología tradicional no se practican, como la creatividad, la crítica constructiva, la capacidad para extraer lo fundamental prescindiendo de lo accesorio, etc.".

"El aprendizaje es mucho más valioso, pues creado por ti mismo, te ayuda a pensar y reflexionar en lo que haces antes de expresarlo o decidir qué evidencias son más representativas para demostrar lo aprendido".

"El portafolio te hace investigar y desarrollar habilidades como la indagación en temas que aún no se dominan".

(Estudiantes de $1^{\circ}$ de Psicopedagogía)

Esta dimensión se consolida como epicentro de las demás, en el sentido de que el estudiante al comenzar a ser consciente de la trascendencia de las competencias en todo su proceso de aprendizaje y posterior integración en el mundo laboral, se responsabiliza de profundizar, relacionar, supervisar, autoevaluar y generar su propio bagaje cultural.

Metodológicamente hablando las competencias son el pilar formativo que más sostén y apuntalamiento pedagógico necesitan, puesto que el único modo de entrenarlas, fijarlas y valorarlas, es a través de la acción. Es decir, a través de metodologías activas, en las que el diseño, reflexión, resolución y valoración de un problema, caso, etc., recaiga en gran medida en el estudiante, bajo la supervisión del docente. Por tanto, la definición de competencias debe ser coherentemente diseñada y vinculada con los objetivos, contenidos y metodología desde el inicio del curso. Al estudiante hay que mostrarle de forma contundente y transparente, cuál es el propósito formativo global de la asignatura, a través de un calendario en el que se describa, qué, cuándo y cómo se desarrollará y relacionará cada tema de la asignatura.

Los comentarios reflexivos de nuestros estudiantes demuestran que esta sistematización en la justificación, organización y desarrollo de la asignatura ha conseguido otro efecto positivo, y es el desarrollo de habilidades metacognitivas de aprendizaje, a través de las cuales el estudiante planifica, profundiza y dirige su propia actividad de manera eficaz. 


\section{Conexión teoría-práctica-aprendizaje}

El portafolio se reconoce como una herramienta útil para relacionar la teoría y la práctica profesional del psicopedagogo. Y es que el hecho de seleccionar una colección de casos reales cercanos a la práctica profesional, a la que tendrán que enfrentarse en el Prácticum del último año de carrera, y cercanos al ámbito laboral que les espera una vez obtenido el titulo académico, motiva y estimula el aprendizaje. En este sentido el portafolio favorece la transferencia entre saberes teóricos (SABER) y saberes prácticos (SABER HACER). Con la ayuda del docente, que adopta el rol de facilitador de evidencias y estándares de resolución óptima y eficaz de problemas, el estudiante encuentra una guía en el camino de búsqueda de respuestas, herramientas, habilidades necesarias para solucionar situaciones comúnmente demandadas en su profesión.

Los siguientes fragmentos extraídos de las reflexiones finales anexadas al final de los portafolios analizados, ilustran estos comentarios:

"Las actividades desarrolladas en el portafolio representan una antesala de los casos a los que nos tendremos que enfrentar profesionalmente, por tanto veo que por fin los trabajos que hay que entregar en una asignatura sirven para algo".

"Las consignas que se nos facilitan en la guía del portafolio, ayudan a relacionar teoría y práctica, porque a través de esta metodología analizo y valoro lo que escribo tal y como lo comprendo, ayudándome de evidencias de búsqueda extraídas de fuentes libremente elegidas".

"Con el portafolio no sólo he conseguido relacionar teoría y práctica, sino que he ampliado los conocimientos que tenía".

(Estudiantes de $1^{\circ}$ de Psicopedagogía)

La facilitación de la búsqueda y contacto con realidades profesionales cercanas a la intervención y orientación psicopedagógica, buscadas por el propio estudiante, en los departamentos de orientación del centro en el que desarrollaron sus estudios o sus anteriores prácticas, en una ONG, en un centro de Ocio y Tiempo Libre, etc., favorece la presentación, justificación y defensa de un contenido teórico de manera original, creativa, realista y útil tanto para sus resultados académicos como para los centros o instituciones con los que ha aprendido a establecer relaciones de cooperación. A su vez, el estudiante pasa a conocer de primera mano las problemáticas, los recursos, las competencias necesarias y el verdadero alcance de sus funciones profesionales, formándose su propia identidad cultural.

\section{Participación}

En este marco, el portafolio se convierte en un gran dinamizador del rol activo no solo del docente, sino del estudiante, puesto que se valora desde el inicio la capacidad para aportar sugerencias e ideas originales, junto con el compromiso en su desarrollo hasta el final tanto autónoma como cooperativamente si las tareas han sido desarrolladas en equipo. Por tanto, se trata de una metodología de enseñanza, aprendizaje y evaluación caracterizada por la flexibilidad y la posibilidad de expresión de los propios valores, iniciativas y concepciones. En definitiva, este proceso de aprendizaje ayuda a plantear al estudiante pautas de actuación profesional de acuerdo a su propia identidad y competencias innatas y adquiridas. 
"El portafolios me ha permitido implicarme más en los trabajos propuestos, sabiendo en todo momento que el producto final iba a ser producto de mi esfuerzo personal".

"El portafolio me ha permitido participar y desarrollar las inquietudes que tenía en relación a esta materia".

"Esta metodología suscita el interés por la búsqueda de fuentes bibliográficas y también por el enriquecimiento tanto personal como académico".

(Estudiantes de $1^{\circ}$ de Psicopedagogía)

Por tanto, la idea que extraen los estudiantes es que resulta necesario que a través de la metodología de la asignatura se provoque interés, inquietud e implicación, aunque ello exija la construcción y elaboración personal de todo un bagaje conceptual e instrumental, que sólo es útil si se consigue relacionar con esquemas conceptuales personales previos y nuevos.

\section{Supervisión y evaluación}

El portafolio plantea un proceso simultáneo de evaluación formativa y continua, que promueve una implicación reflexiva y una autorregulación del propio rol, de todas las audiencias protagonistas del proceso de aprendizaje. Como afirma Klenovski (2004), para el desarrollo de un portafolio es crucial el pensamiento y la práctica reflexiva. Todo ello supone para el docente un trabajo extraordinario de feed-back y orientación inmediata, que una vez recibido por el estudiante pasa a convertirse en un deber de auto-supervisión y mejora del producto primigenio. El proceso culmina cuando el estudiante es capaz de justificar y argumentar, reflexiva, crítica y creativamente su producto, de forma que se le deja también tiempo y espacio para que aprenda de sus errores e imprima a sus resultados un carácter personal, dinámico y novedoso.

"El feed-back que he recibido de la profesora supone una gratificación inmediata de mi trabajo muy positiva y estimulante".

"La evaluación propuesta me permite determinar en parte la nota que me van a asignar, si ésta se formula en función de las evidencias que presento en el portafolio".

"Esta metodología te ayuda a saber lo que tienes que corregir y potencia una mejora continua".

"Los criterios de evaluación están claros de antemano, he sabido qué y cómo iba a ser evaluado desde el principio".

(Estudiantes de $1^{\circ}$ de Psicopedagogía)

Algunas experiencias desarrolladas con el portafolio en otras universidades españolas muestran el potencial de esta herramienta como instrumento de evaluación (Hernández, 2004) y como vía para estimular la experimentación, reflexión e investigación (Lyons, 1999).

\section{CONCLUSIONES}

A la hora de presentar las conclusiones optamos por responder, a partir de la información obtenida en el análisis de los datos, a las hipótesis que formulamos inicialmente en el planteamiento del trabajo. 
$1^{\circ}$. Los alumnos de "Diagnóstico en Educación" de la titulación de Psicopedagogía perciben que su rendimiento mejora con la práctica del Portafolio, pese a que no desestiman totalmente las posibilidades del aprendizaje tradicional en la consecución de determinadas habilidades como la iniciativa, la síntesis y la autonomía.

$2^{\circ}$. Se puede interpretar que las relaciones interpersonales establecidas entre los estudiantes resultan más estables, profundas y comprometidas en el portafolio. Así mismo las relaciones entre el profesorado y los estudiantes son más cercanas, estimulantes y enriquecedoras para ambos. Se percibe una implicación activa por ambas partes que estimula la participación continua y el enriquecimiento mutuo.

$3^{\circ}$. El grado de aprendizaje significativo que manifiestan tener estos estudiantes tras el desarrollo del portafolio es mayor que el reconocido durante el desarrollo de tareas más tradicionales. Afirman que la búsqueda, el repaso, la elaboración y la exposición de actividades al resto de sus compañeros y al docente les facilita la comprensión y fijación de los contenidos de la asignatura.

$4^{\circ}$. El alumnado reconoce que con esta metodología es mucho más complicado autoplanificarse sin entrenamiento previo, puesto que durante los años académicos anteriores no se encontraron en la tesitura de tener que organizar su tiempo conjuntamente con compañeros, profesorado e individualmente y coordinadamente con otras asignaturas de la titulación, puesto que el peso específico de la misma corre a cargo no solo del profesor responsable sino de forma compartida junto con el alumnado.

De todo lo expuesto, concluimos que los hallazgos de la investigación indican que el portafolio es una herramienta eficaz para el aprendizaje en asignaturas inicialmente planificadas para desarrollar exclusivamente en el aula. Ahora, a través de su aplicación teórico práctica, los estudiantes desarrollan diversidad de habilidades que les capacitan para construir conocimiento y una identidad cultural personalizada, a la vez que aprenden a responsabilizarse de su propio aprendizaje y a ser conscientes de los procesos metacognitivos empleados. Por tanto, se erige como una herramienta pedagógica que se ajusta con coherencia y eficacia a los preceptos del Espacio Europeo de Educación Superior.

\section{BIBLIOGRAFÍA}

Alonso, C.M. (1992) Estilos de aprendizaje: análisis y diagnóstico en estudiantes universitarios. Madrid: Universidad Complutense.

Aronson, E. (1978) The Jigsaw Classroom. Beberly Hills, California: Sage.

Colás, Ma.P. y De Pablos, J. (2005) La universidad en la unión europea. El espacio europeo de educación superior y su impacto en la docencia. Málaga: Aljibe.

Cowan, J. (1998) On Becoming an Innovative University Teacher: Reflection in Action. Open University Press.

De la Cruz, A. (1999) Formación del profesor universitario en metodología docente. En Ruiz, J. (coord) Aprender y enseñar en la Universidad. Iniciación a la docencia universitaria. Jaén: Universidad de Jaén.

Duda, C.M. y Rely, P. (1990) Learning Styles. Nancy: Press Universitaire Nancy. 
De Miguel, M. (coord.) (2006) Metodologías de enseñanza y aprendizaje para el desarrollo de competencias. Orientaciones para el profesorado universitario ante el EEES. Colección Editorial. Madrid: Alianza.

Hernández, N. (2004) La evaluación mediante portafolios. En Rodríguez, R., Hernández, J. Y Fernández, S. (Coords) Relaciones económicas internacionales. Docencia universitaria, orientaciones para la formación del profesorado. Oviedo: Universidad de Oviedo.

Johnson, D.W. y Johnson, R.T. (1989) Cooperative Learning: What Special Education Teachers Need to Know. Pointer, 33 (2), 5-10.

Klenowski, V. (2004) Desarrollo del portafolios. Madrid: Narcea.

González, J. y Wagennar, R. (coords.) (2003): Tuning Educational Structures in Europe. Deusto: Universidad de Deusto.

Lyons, N. (1995) Teacher Portfolio Projet. Gorham: University of Southern Maine.

Lyons, N. (1999) El uso del portafolios para el aprendizaje y la evaluación. Buenos Aires: Amorrortu.

Marcelo, C. (2001) Función docente: nuevas demandas para viejos propósitos. En Marcelo, C. (coord.) La función docente. Madrid: Síntesis.

Mayor, C.M. (coord.) (2003) Enseñanza y aprendizaje en la Educación Superior. Barcelona: Octaedro-EUB.

Monereo, C. y Pozo, J.I. (eds.) (2003) La universidad ante la nueva cultura educativa. Madrid: Síntesis.

Sharan, S. (1980) Cooperative learning in teams: recent methods and effects on achievement attitudes and athic relations. Review of Educational Researcho, 50. 241 271.

Shores, E.F. y Grace, C. (1998) El Portafolio paso a paso. Biblioteca Infantil. Barcelona: Graó.

Torbay, A. Muñoz de Bustillo, M.C. y Hernández, C. (2001) Los estudiantes universitarios de carreras asistenciales: Qué habilidades interpersonales dominan y cuáles creen necesarias para su futuro profesional. Revista Aula Abierta, 78, 1-17.

Zabalza, M.A. (2000) La enseñanza universitaria: el escenario y los protagonistas. Madrid: Narcea. 